\title{
Sum-Frequency Generation of High-Energy and High-Beam-Quality Ultraviolet Pulses
}

\author{
Oystein Farsund and Gunnar Rustad \\ Land and Air Systems Division, Norwegian Defence Research Establishment (FFI), P.O. Box 25, 2027 Kjeller, Norway
}

Correspondence should be addressed to Oystein Farsund, oystein.farsund@ffi.no

Received 1 July 2011; Accepted 24 August 2011

Academic Editor: Alexandre Kudlinski

Copyright ( $) 2011$ O. Farsund and G. Rustad. This is an open access article distributed under the Creative Commons Attribution License, which permits unrestricted use, distribution, and reproduction in any medium, provided the original work is properly cited.

Sum-frequency generation of UV pulses exceeding $25 \mathrm{~mJ}$ and with beam quality $M^{2} \sim 5$ has been demonstrated by mixing the third harmonic pulses of a flash lamp pumped $1.06 \mu \mathrm{m}$ Nd:YAG laser with $1.7 \mu \mathrm{m}$ pulses from an optical parametric oscillator pumped by the same laser in a compact setup.

\section{Introduction}

High-energy ultraviolet (UV) pulses with high beam quality have important lidar applications, such as in standoff detection of biological aerosols and in ozone detection (see, e.g., [1]). While the third (355 nm) and fourth $(266 \mathrm{~nm})$ harmonics of the $\mathrm{Nd}$ :YAG laser can be used in some applications, the optimal laser wavelength in the above-mentioned applications is close to $290 \mathrm{~nm}$. These applications also demand portable instruments suitable for outdoor experiments and with good beam quality. The topic of this work is generation of high-energy pulses with good beam quality in the 290$300 \mathrm{~nm}$ range from a compact setup.

\section{Sum-Frequency Generation (SFG) Architecture}

There are several ways to reach wavelengths around $300 \mathrm{~nm}$ starting out with a single Nd:YAG laser, discussed and demonstrated experimentally for selected UV wavelength ranges by Fix and Ehret [2]. They achieved up to $20 \mathrm{~mJ}$ at $320 \mathrm{~nm}$ by summing $532 \mathrm{~nm}$ radiation with an OPO pumped by $532 \mathrm{~nm}$, starting out with $410 \mathrm{~mJ}$ at $1064 \mathrm{~nm}$. The beam quality was not reported; however, the divergence was measured to $0.7 \mathrm{mrad}$. In our work, $\sim 290 \mathrm{~nm}$ is reached by mixing the laser third harmonic with the signal from an optical parametric oscillator (OPO) pumped by the laser's fundamental wavelength in a sum-frequency generation (SFG) process. The output UV wavelength can be varied by tuning the OPO wavelength (and with subsequent tuning of the SFG crystal), but this has not been demonstrated in this work.

Efficient conversion in the SFG stage requires the interacting beams to overlap well both temporally and spatially, and beam divergences smaller than the acceptance angle of the nonlinear interaction. Temporal overlap is ensured by pulse timing and similar pulse lengths. High beam qualities of the interacting beams assure sufficiently low beam divergence in combination with suitable diameters as well as even intensity distributions, which allow for optimal spatial overlap. These matters were discussed in detail in [3], and a remarkable conversion efficiency was demonstrated, however, at the cost of a more complex setup than was the aim of our work. The quality of the UV beam was not reported; however, a far field plot and given beam diameter indicate a UV beam with $\sim 3 \mathrm{~mm} \cdot \mathrm{mrad}$ at $320 \mathrm{~nm}$, and pulse energy more than $100 \mathrm{~mJ}$.

\section{Setup}

The experimental setup is depicted and sketched in Figure 1. A flash lamp pumped Nd:YAG laser (Quantel Brilliant B) with $5 \mathrm{~ns}$ pulse length (FWHM) was used as laser source in our experiments. The laser was operated in multilongitudinal mode in the experiments. A thin film polarizer located 


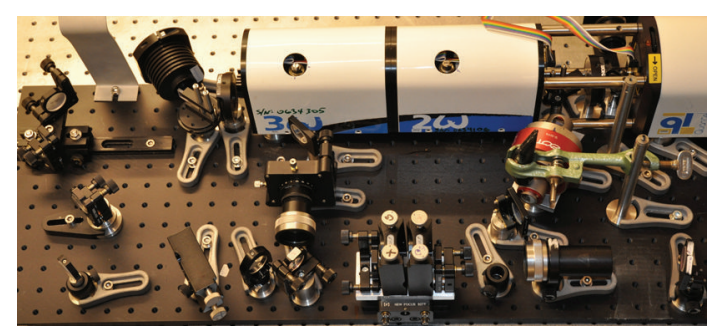

(a)

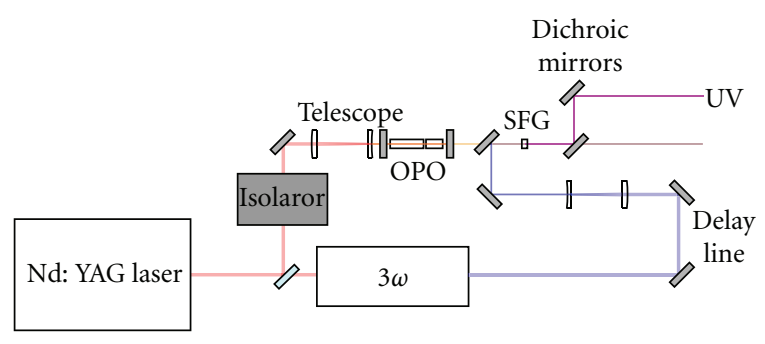

(b)

FIgUre 1: Top view of the experimental setup, depicted (a) and sketched (b). An adequate portion of the pulse energy was split off from the Nd:YAG-laser for OPO pumping. The residual energy generated $3 \omega$ using the commercial harmonic generator modules. The OPO signal and $3 \omega$ beams were combined in front of the SFG stage.

just after the exit port of the laser cleaned up the polarization state of the pump beam. A beam with adjustable energy was picked off the laser beam by a half-wave plate and another polarizer. This beam was used to pump the OPO, which is described in Section 3.1. The remaining laser energy was passed to the second and third harmonic $(3 \omega)$ stages (also from Quantel). The two-pass-pumped OPO required an isolator at the pump wavelength to block reflection back to the laser. The pump beam diameter was reduced to $\sim 4 \mathrm{~mm}$ by the use of a Galilean telescope (a planoconvex lens, $f=$ $125 \mathrm{~mm}$ succeeded by a planoconcave lens, $f=-75 \mathrm{~mm}$ ). Similarly, a half-wave plate and a thin film polarizer were used to control the $3 \omega$ pulse energy, and a Galilean telescope consisting of UV-grade fused silica lenses coated for UV reduced the $3 \omega$ beam to approximately the same diameter as the OPO signal beam. Two mirrors allowed the adjustment of spatial overlap (of position and direction) between the $3 \omega$ and IR beams. The path length for the $3 \omega$ beam could be changed for optimal temporal overlap, and the delay was optimized for maximum output energy by varying the path length during operation of the SFG.

3.1. OPO with Orthogonal Critical Planes. We have previously shown that good beam quality can be obtained from a pulsed OPO if the beam size is small enough that the Fresnel number of the resonator is smaller than the number of roundtrips during the pump pulse [4]:

$$
W \leq \sqrt{\frac{\lambda L_{r} N_{\mathrm{rt}}}{n_{r}}}=\sqrt{\frac{\lambda t_{p} c}{2 n_{r}^{2}}},
$$

where $W$ is the beam radius, $\lambda$ is the resonated wavelength, $L_{r}$ is the mirror spacing, $N_{\mathrm{rt}}$ is the number of resonator round trips during a pump pulse, $t_{p}$ is the pump pulse length, $c$ is the speed of light in vacuum, and $n_{r}$ is the average refractive index in the resonator. For a $5 \mathrm{~ns}$ pump pulse length and an average refractive index of 1.5, the maximum beam radius is $\sim 0.7 \mathrm{~mm}$. If we restrict the incident pump fluence to $1 \mathrm{~J} / \mathrm{cm}^{2}$ to avoid damage, (1) then limits the maximum pump energy to $\sim 8 \mathrm{~mJ}$. In the high-pulse-energy case studied in this work, the beams need to be significantly larger than the $0.7 \mathrm{~mm}$ limit to avoid optical damage, thus other mechanisms are needed to ensure good beam quality.

When using critical type 2 phase matching, the angular spectrum (and hence the beam quality) in the critical direction in the OPO is improved by the limited acceptance angle of the nonlinear interaction. However, since this restriction only occurs in the critical direction, the beams from such OPOs with beams larger than given by (1) often suffer from highly asymmetric beam qualities [5]. Recently, we demonstrated a method to improve the beam quality and symmetry from OPOs with large beams using two different crystals with orthogonal critical planes in the same OPO [6]. By carefully selecting the two nonlinear crystals, narrow acceptance angles in both directions make the far field of the OPO beam symmetrical.

This approach has been used in this work, where the OPO contained one $30 \mathrm{~mm}$ long KTP crystal and one $20 \mathrm{~mm}$ long $\mathrm{BBO}$ crystal, both AR coated and cut for type 2 phase matching of $1.064 \mu \mathrm{m}$ pumped conversion to $1.7 \mu \mathrm{m}$. The crystals had $8 \mathrm{~mm} \times 8 \mathrm{~mm}$ apertures, and the pump beam was double passed. A significant part of the $2.84 \mu \mathrm{m}$ idler wave is absorbed in BBO in this OPO, but as we have previously shown, this idler absorption can actually lead to improved OPO performance for low-pulse-rate OPOs, mainly owing to reduced back conversion in the OPO [4].

In order to reduce the risk of optical damage, the incident pump fluence was restricted to approximately $1.5 \mathrm{~J} / \mathrm{cm}^{2}$, corresponding to $120 \mathrm{~mJ}$ pump energy with the given beam diameter $\left(3.6 \mathrm{~mm}\right.$ at $1 / e^{2}$ level $)$ and $50 \%$ signal output coupling. The OPO was optimized with respect to signal output energy and beam quality, which was measured to $\left(M_{x}^{2}, M_{y}^{2}\right)=$ $(1.6,2.4)$. The OPO signal energy versus pump energy is shown in Figure 2.

3.2. SFG Stage. Based on simulations of the SFG stage with parameters as described above, a $5 \mathrm{~mm}$ long BBO crystal for collinear critical type I phase matching was selected. The crystal was AR coated at $355 \mathrm{~nm}$ and $1.7 \mu \mathrm{m}$ on the input face, and AR coated around $295 \mathrm{~nm}$ on the output face. The generated UV beam was separated from the depleted pump beams by means of two dichroic mirrors, and the pulse energies were measured using an Ophir power meter equipped with a 10-AP detector. The near and far fields of the interacting beams were measured with a Spiricon III Pyrocam, the far field in the focal plane of a lens with $2 \mathrm{~m}$ focal length. 


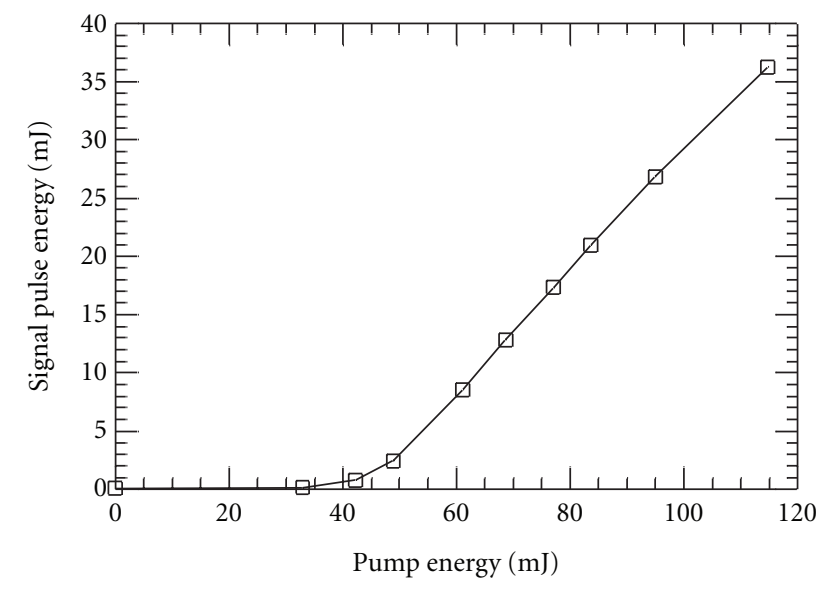

FIGURE 2: Measured OPO signal energy as function of pump energy.

\section{SFG Experimental Results}

The generated UV pulse energy was measured as function of the $3 \omega$ pulse energy while the $1.7 \mu \mathrm{m}$ pump from the OPO was kept constant. This was repeated for different OPO signal energy levels. The results are summarized in Figure 3, from which we notice that increasing the $1.7 \mu \mathrm{m}$ pump energy from $12 \mathrm{~mJ}$ to $26 \mathrm{~mJ}$ increased the UV pulse energy but also that a further increase from $26 \mathrm{~mJ}$ to $34 \mathrm{~mJ}$ gave only marginally increased output energy, which is mainly attributed to the higher $3 \omega$ pulse energy.

The near and far fields of the laser fundamental, OPO signal, $3 \omega$ and UV beams were recorded for the purpose of beam quality measurements. Measured near and far field fluence profiles are shown in Figure 4.

Before calculating the $\left(M_{x}^{2}, M_{y}^{2}\right)$ values as given in Table 1, the background was measured and subtracted from the signal. For comparison with other work, we also estimated the diameter-divergence product of the generated UV beam. This was done using the $10 \%-90 \%$ knife-edge values of the measured images in the $x$ - and $y$-directions. This was found to be $0.76 \mathrm{~mm} \cdot \mathrm{mrad}$ in the $x$-direction and $1.2 \mathrm{~mm} \cdot \mathrm{mrad}$ in the $y$-direction.

\section{Discussion}

For a given crystal, local (at a specific point in the beam and a specific time) SFG conversion efficiency depends on the intensity of both incident beams. For $100 \%$ conversion, the flux densities of photons must match so that no photons are left over in either beam, and the intensities must be such that there is full conversion at the output end of the crystal. If the intensities are too high, there will be back conversion, which reduces both efficiency and beam quality. High total conversion efficiency requires spatial and temporal overlap of the beams and that each beam has uniform intensity in time and space.

In our experiment, the pulse lengths of the interacting beams are similar. However, as the laser operates in multilongitudinal mode, there will be random spikes on the laser

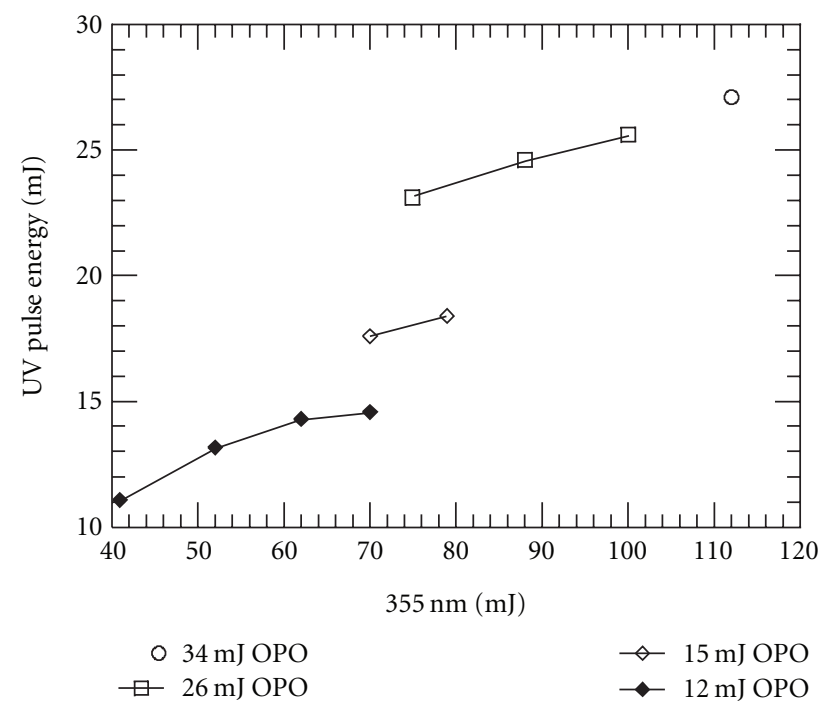

FIGURE 3: Measured energy of the generated UV pulse for different OPO signal and $3 \omega$ pulse energies.

TABLE 1: Calculated $\left(M_{x}^{2}, M_{y}^{2}\right)$ values (dimensionless) based on measured images of near and far fields.

\begin{tabular}{lll}
\hline Beam & $M_{x}^{2}$ & $M_{y}^{2}$ \\
\hline Laser fundamental & 2.1 & 2.4 \\
OPO signal $(\sim 8 \mathrm{~mJ})$ & 1.6 & 2.4 \\
$3 \omega$ & 3.3 & 4.7 \\
SFG $(\sim 13 \mathrm{~mJ})$ & 4.2 & 5.2 \\
\hline
\end{tabular}

pulse, which will be preserved through the harmonic stages. The OPO signal will also get spikes, but because of the OPO resonator and the nonlinear generation process they will in general not preserve the spike pattern of the pump pulse. Thus, both pulses to the SFG stage have temporal spikes which in general do not overlap, and this reduces conversion efficiency. It should be noted that even if the spikes should overlap, their high intensity might cause back conversion and thus both reduced conversion efficiency and beam quality.

Another limiting factor for the conversion efficiency in our experiment is the nonuniform spatial distributions of the $355 \mathrm{~nm}$ and $1.7 \mu \mathrm{m}$ beams and hence their imperfect spatial overlap. The fluence profile measurements in Figure 4 show the intensity integrated over the entire pulse, so the real mismatch and nonuniformity may be worse if the spatial distribution varies during the pulse. Our laser has a variable reflectivity mirror as output coupler, and it has been shown that such lasers tend to produce beams with time-dependent transverse distribution [7].

For all these reasons, it is predicted that a significant improvement of the efficiency of the SFG process can be obtained with a single-longitudinal mode pump laser and more uniform transversal profiles of the beams incident on the SFG stage. 


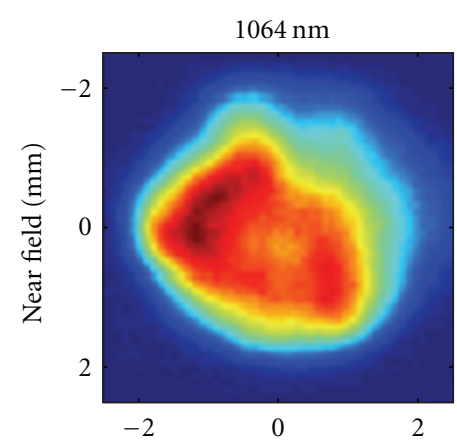

(a)

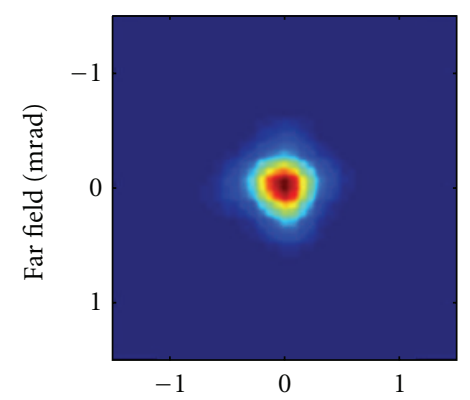

(e)

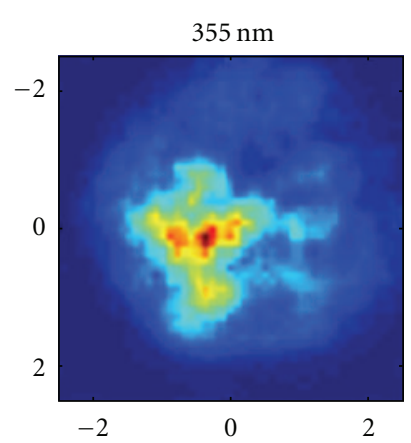

(b)

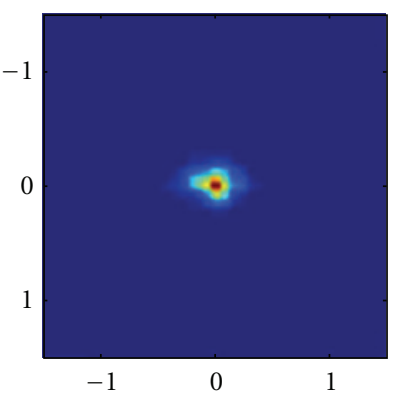

(f)

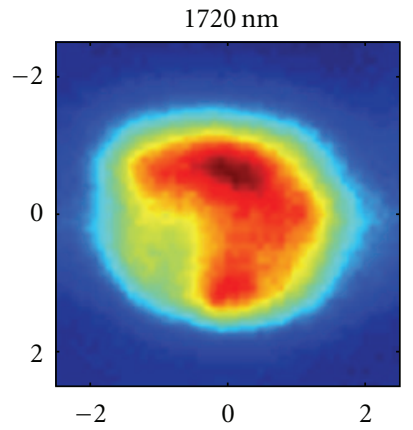

(c)

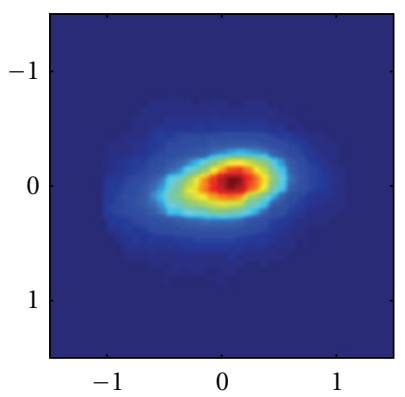

(g)

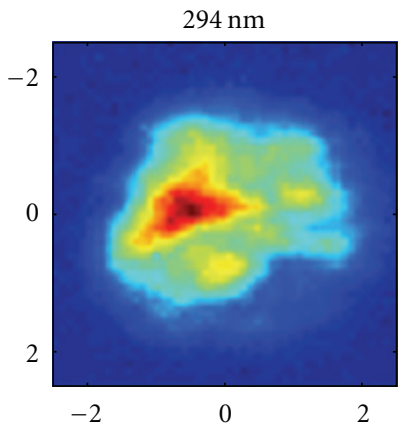

(d)

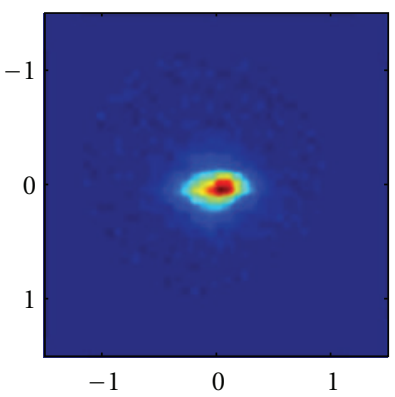

(h)

Figure 4: Near- ((a)-(d)) and far-field fluence profiles ((e)-(h)) for a single pulse of the laser fundamental, $3 \omega$, OPO signal, and generated UV beams.

\section{Conclusion}

In conclusion, we have experimentally demonstrated nanosecond pulses at $294 \mathrm{~nm}$ with energy exceeding $25 \mathrm{~mJ}$ and beam quality $M^{2} \approx 5$ using a single Nd:YAG laser as pump source in a compact setup. The third harmonic of the laser was sum-frequency mixed with the signal from an OPO pumped by the laser fundamental, generating UV pulses in a critical type I collinearly phase matched process. A key to the good beam quality obtained here is the high beam quality of the beam from the OPO. This was obtained using two crystals with orthogonal critical planes in the OPO [6].

\section{References}

[1] J. R. Lakowicz, "Protein fluorescence," in Principles of Fluorescence Spectroscopy, pp. 446-487, Kluwer Academic/Plenum Publishers, New York, NY, USA, 2nd edition, 1999.

[2] A. Fix and G. Ehret, "Intracavity frequency mixing in pulsed optical parametric oscillators for the efficient generation of continuously tunable ultraviolet radiation," Applied Physics B, vol. 67, no. 3, pp. 331-338, 1998.

[3] D. J. Armstrong and A. V. Smith, "All solid-state high-efficiency tunable UV source for airborne or satellite-based ozone DIAL systems," IEEE Journal on Selected Topics in Quantum Electronics, vol. 13, no. 3, pp. 721-731, 2007.

[4] G. Rustad, G. Arisholm, and Ø. Farsund, "Effect of idler absorption in pulsed optical parametric oscillators," Optics Express, vol. 19, no. 3, pp. 2815-2830, 2011.
[5] A. V. Smith and M. S. Bowers, "Image-rotating cavity designs for improved beam quality in nanosecond optical parametric oscillators," Journal of the Optical Society of America B, vol. 18, no. 5, pp. 706-713, 2001.

[6] Ø. Farsund, G. Arisholm, and G. Rustad, "Improved beam quality from a high energy optical parametric oscillator using crystals with orthogonal critical planes," Optics Express, vol. 18, no. 9, pp. 9229-9235, 2010.

[7] G. Anstett and R. Wallenstein, "Experimental investigation of the spectro-temporal dynamics of the light pulses of Qswitched Nd:YAG lasers and nanosecond optical parametric oscillators," Applied Physics B, vol. 79, no. 7, pp. 827-836, 2004. 

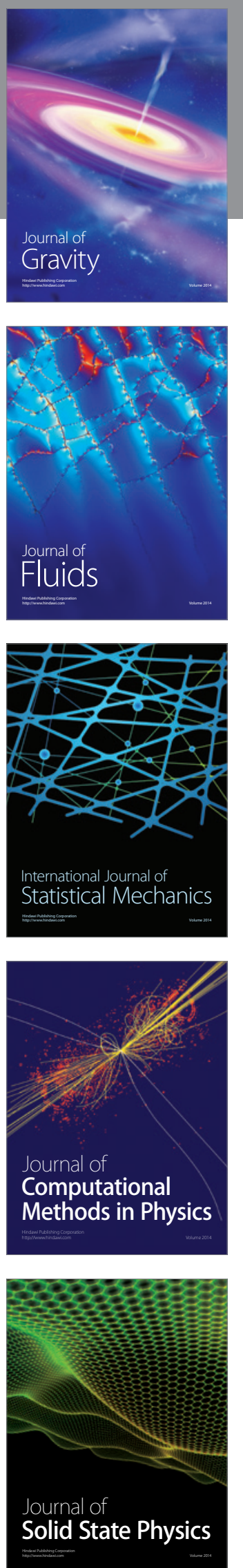

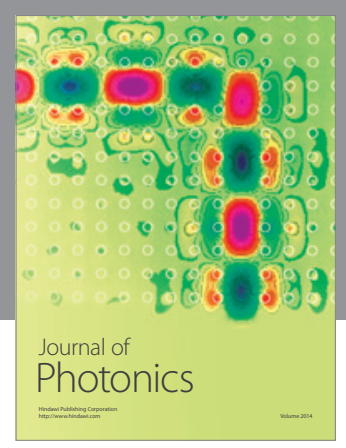

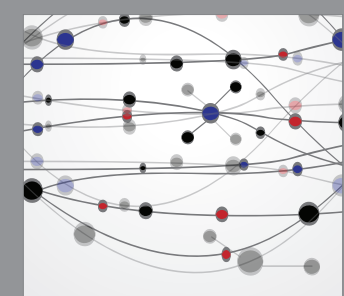

The Scientific World Journal
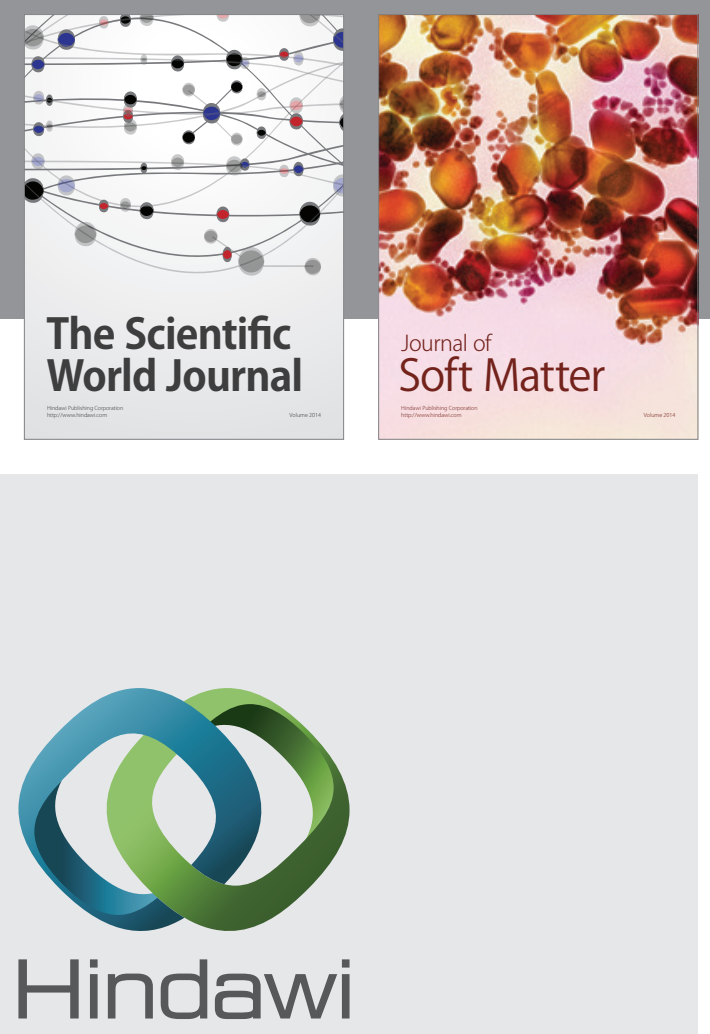

Submit your manuscripts at

http://www.hindawi.com
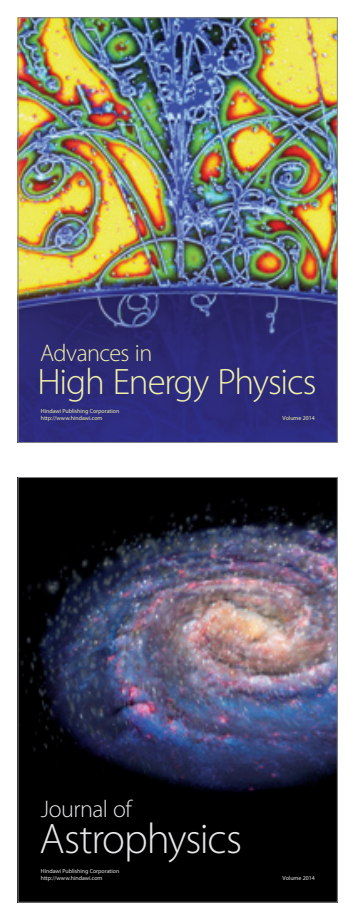
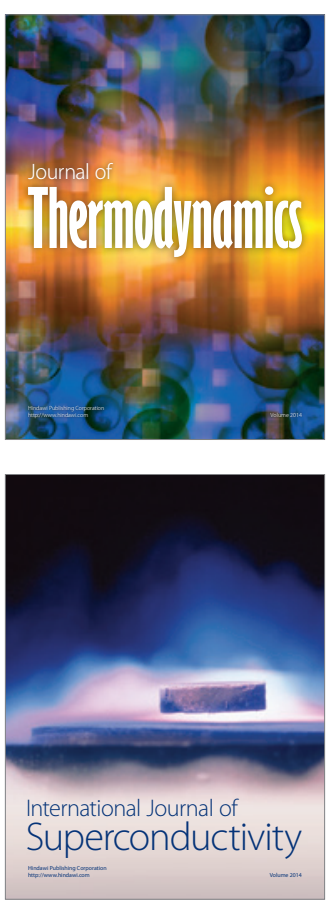
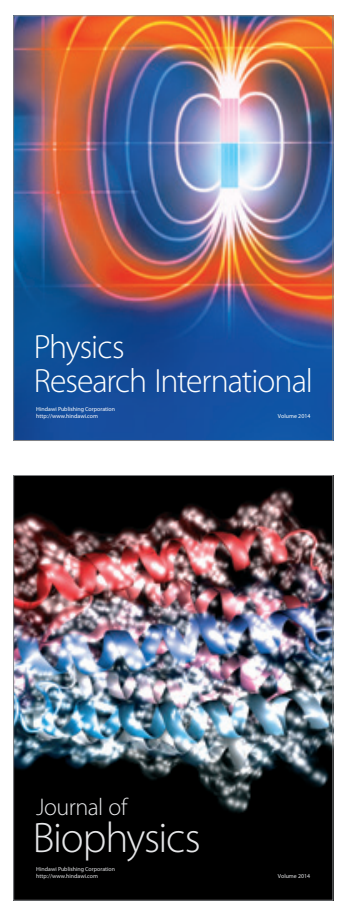
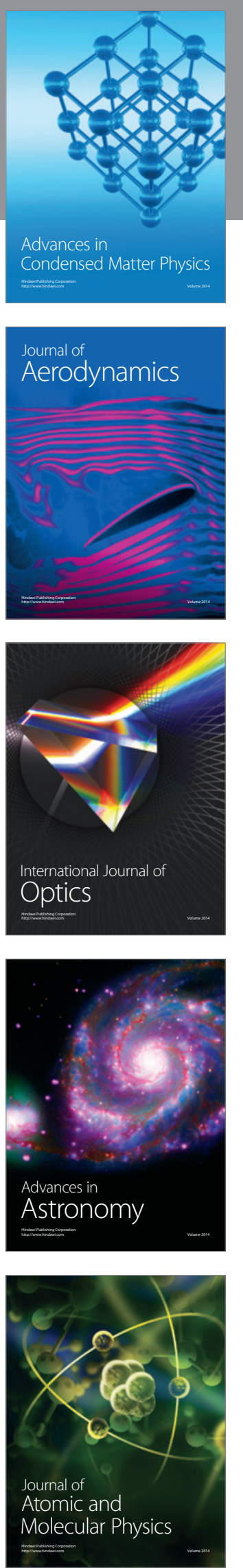